\title{
VIII.
}

\section{Ueber Missbildungen am Ohr und im Bereiche des ersten Kiemenbogens.}

Von Rud. Virchow.

(Hierzu Taf. VIl. Fig. $\check{\jmath}-7$ )

\begin{abstract}
Die Mittheilungen über. Halskiemenfisteln beim Menschen, welche der bochverdiente Veteran der Pathologie, Heusinger, vor Kurzem veröffentlichte, insbesondere der zweite von ihm beobachtete Fall (dieses Archiv Bd. XXIX. S. 361 Taf. XII. Fig. 2-3) brachten mir einige Erfahrungen über rerwandte Missbildungen in die Erinnerung, welche der weiteren Aufmerksamkeit der Beobachter empfohlen zu werden verdienen.

Heusinger fand bei einem 7 jährigen, mit ejgenthümlichen Störungen des. Gehörs behafteten Mädchen ausser der angebornen Halsfistel an beiden äusseren Ohren Missbildungen, nämlich Erbabenheiten, Löchelchen und Narben, welche einer frühen Entwickelungszeit angehören müssen. Er bezieht sie, gewiss mit Recht, auf eine unregelmässige Schliessung der Ohrkiemenspalten und er erinnert, unter Beifügung einer anderen Beobachtung, an die Halslappen der Ziegen.

Sieht man von der Halsfistel ab, deren Zusammentreffen mit den zuletzt genannten Missbildungen, wenn auch nicht zufällig, doch jedenfalls nicht beständig ist, so finden sich in der Literatur manche Angaben, welche darthun, dass nicht ganz selten Missbildungen des äusseren Ohres mit ausgedehnteren Störungen in der Bildung der zum ersten Kiemenbogen gehörigen Theile zusammenfallen. Am häufigsten ist Hasenscharte und Wolfsrachen beobachtet worden. Freilich gibt es auch Fälle, wo alle anderen Theile normal gebildet zu sein und die Störungen des äusseren Ohres allein für sich zu bestehen scheinen.
\end{abstract}


Schon Voigtel (Handb. der path. Anat. Halle 1804. Bd. II. S. 34-35) hat eine Zusammenstellung der inm bekannten Fälle geliefert. Es handelte sich dabei theils um vollständigen oder theilweisen Mangel des äusseren Ohres, theils um widernatürliche Ansatzstellen, theils um überzählige Bildungen und Spalten des äusseren Ohres. Lassen wir die ersteren Fälle zunächst ausser Betracht, so bleiben folgende interessante Abweichungen: 1) die Ohren sassen am Halse (Sebenicius), 2) ein Ohr sass auf der Schulter (Wolf), 3) ein Ohr sass mitten auf der Backe und um dasselbe standen verschiedene kleine warzenartige Auswücbse (Fielitz), 4) unter dem einen 0 hr befand sich noch ein zweites halbes Ohr (Stark), 5) nahe am Ohr auf der Backe sass noch ein grosses Ohrläppchen (Stark), 6) das Ohr war quer durchgespalten und die Hälften einen Finger breit von einander abstehend (Löffler).

Diese Fälle umfassen so ziemlich die verschiedenen Möglichkeiten, welche auch von späteren Beobachtern aufgezeichnet worden sind. Erwägt man nun, dass das äussere $0 \mathrm{hr}$ nebst dem knorpeligen Gehörgange sich aus der Haut entwickelt, welche die aussere Oeffnung der früheren Kiemenspalte begrenzt, und dass die Ohrmuschel schon am Ende des dritten Monats in allen Theilen da ist (Bischoff Entwickelungsgeschichte S. 409, Kölliker Entwickelungsgeschichte S. 324), so wird man in allen diesen Fällen folgern müssen, dass es sich um sehr frühe Störungen in der Schliessung der ersten Kiemenspalte handelt. Schon A. G. H. Birnbaum (Beschreibung und Kritik einer eigenthümlichen Bildungshemmung. Inaug. Diss. Giessen 1848. S. 15) hat diess in einer, unter der Leitung $\mathrm{Th}$. Bischoff's gearbeiteten Abhandlung genau entwickelt. Es liegt ferner nahe, dass in manchen Fällen nur eine auf die Haut und die aus ihr hervorgebenden Gebilde beschränkte Störung besteht, während in anderen die benachbarten Kiemenbogen und die daraus hervorgehenden Gebilde an derselben Antheil nehmen. In Beziehung auf diese letzteren ist $z u$ erinnern, dass der erste Kiemenbogen nach der Entdeckung von Reichert den Hammer und Amboss liefert, dass der Hammer ursprünglich mit dem Meckel'schen Fortsatz zusam- 
menhängt, an welchem sich der Unterkiefer entwickelt, dass ferner dieser Kiemenbogen ausser dem Unterkieferfortsatz noch einen zweiten, den Oberkieferfortsatz, hervortreibt, welcher das Flügelbein (Lamina interna ossis pterygoidis) und das Gaumenbein liefert. Aus dern zweiten Kiemenbogen werden der Steigbügel, der Griffelfortsatz und das kleine Horn des Zungenbeins, aus dem dritten das Zungenbein selbst mit den grossen Hörnern (Kölliker ebend. S. 214-220). Es versteht sich daher von selbst, dass je nach Umständen mit der Missbildung des äusseren Ohres Missbildungen des Gebörganges, der Gehörknöchelchen, des Unterkiefers und wenigstens gewisser Theile des Oberkiefers, des Gaumen- und Flügelbeines, sowie Abweichungen am Halse zusammenfallen können.

Lucae hat neulich eine Reihe solcher Fälle, theils nach eigenen, theils nach fremden Untersuchungen zusammengestellt (Archiv Bd. XXIX. S. 62 folg.), und namentlich auf die schönen Beobachtungen von A. Thoms on (Edinb. monthly Journ. 1847. April.) hingewiesen. Ohne auf die genetischen Verbältnisse einzugehen, fand dieser sorgfältige Beobachter folgende thatsächlichen Zustände in Fällen von Missbildung des äusseren Ohres: 1) unvollständige Entwickelung des cutanen Theils des Apparates, nämlich des äusseren Ohres und des äusseren Theils des Gehörganges, 2) Fehlen des Trommelfells, des Annulus tympanicus und des knöchernen Theils des Gehörganges, in Folge der unvollständigen Entwickelung des 0 s tympanicum oder eines Theils des Gebildes, welches bei niederen Thieren diesen Namen trägt, 3) inangelhaften Zustand der Trommelhöhle und der Kette der Gehörknöcbelchen, 4) gelegentliche Unregelmässigkeit oder Mangel in der Entwickelung der malaren, palatinen und maxillaren Abschnitte des Gesiehts. In Beziehung auf die Punkte 2 und 4 erwähne ich noch besonders, dass Thomson in 2 Fällen die Anlage einer Gaumenspalte (einmal war nur die Uvula gespalten) und Fehlen oder Unregelmässigkeit der oberen seitlichen Schneidezähne fand; in einem dritten lag der Oberkiefer so hoch und weit zurück an die Seite der Wange gedrängt, dass der Joehbogen zu fehlen schien; zugleich konnte der Proc. auditorius oss. temporum nicht durcbgefüblt werden und links schien der Gelenkhöcker zu fehlen, so 
dass der Kopf des Unterkiefers beträchtlich an der Wange vorsprang.

Es erhellt aus diesen Beobachtungen einerseits der Zusammenhang gewisser Störungen in der Bildung des Ohres mit denen genetisch zusammenhängender Theile des Gesichts, worauf in Beziehung auf die Gebörknöchelchen schon Wallmann (dies. Archiv Bd. XI. S. 508) hingewiesen hat, andererseits die grosse prakische Bedeutung jener Störungen. Was mich aber vor Allem bestimmt, die Aufmerksamkeit darauf zu lenken, das ist die Ueberzeugung, dass sich bestimmte Anbaltspunkte für die Pathogenie nicht bloss dieser combinirten Störungen, sondern auch der einzelnen von ihnen daraus gewinnen lassen, und dass schon desshalb auch geringere Grade der Veränderung ein besonderes Interesse besitzen.

Als geringster Grad darf' wohl der Fall bezeichnet werden, wo scheinbar nichts, als gewisse überzählige Theile des äusseren 0 hres neben demselben vorkommen. Man hat diese gelegentlich als accessorische oder supernumeräre Auswüchse, Ohrläppchen oder Ohrklappen bezeichnet. Dahin gehört der eine schon erwähnte Fall von Stark; ferner einer von Middeldorpf (die Galvanokaustik S. 128). Ich selbst kenne einen sonst wohlgebildeten Knaben, der einen cylindrischen Auswuchs von etwas über $\frac{1}{2}$ Cent. Länge gerade am unteren Ende des einen Ohrläppchens trägt; auch habe ich einnial einen solchen Auswuchs untersucht, den Dr. Friedberg exstirpirt hatte. Die Structur dieser Auswüchse (Auricularanhänge) ist ziemlich constant und sie unterscheidet sich, wie es scheint, in nichts von der Structur derjenigen, welche sich bei complicirten Störungen finden. Eine solche hat früer Max Schultze (Archiv Bd.XX. S. 378, Taf. XI. Fig. 1) beschrieben.

Die Auricularanhänge bestehen aus Haut, Unterhaut und Knorpel. Die Haut ist in der Regel glatt, zuweilen stark mit feinen Haaren besetzt; darunter liegt ein manchmal sehr mächtiges und mässig gefässreiches Fettpolster; zu innerst findet sich Netzknorpel in Form eines soliden Zapfens oder einer zungenförmigen Platte oder eines rundlichen Kornes, Der Knorpel reicht ziemlich tief 
in das unterliegende Gewebe hinein, ohne jedoch in der Regel mit dem 0hrknorpel in continuirlicher Verbindung zu stehen. Die Auricularanhänge gleichen jedoch im Grossen unzweifelhaft am meisten dem Ohrläppchen, und namentlich die kleineren, welehe nur sehr wenig oder gar keinen Knorpel enthalten, fühlen sich eben so weich, ich möchte sagen, lipomähnlich an, wie der genannte Theil.

Allein, abgesehen von dem einen, vorbin erwähnten und einem anderen, noch zu beschreibenden Falle, fand ich sie stets an anderen Stellen, als am Läppchen. Am häufigsten sitzen sie vor dem Ohr, in der Gegend des Tragus oder neben der Incisura intertragica, manchmal näher, manchmal ferner. Je complicirter die Störung ist, um so mehr können sie sich von dem Ohr entfernen, wie wir späler noch genauer ausfuhren werden. Manchmal ist es nur ein einziger, der jedoch wieder lappig oder ästig sein kann, in der Regel aber sind es mehrere (Taf. VIl. Fig. 5-6). Besonders häufig fand ich 3, über einander sitzende, so dass eine gewisse Aehnlichkeit mit der Anlage der ursprünglichen über einander liegenden Kiemenbogen entsteht. Dabei ist ihre Grösse sehr veränderlich; wenn es mehrere sind, so pflegen kleinere und grössere zu wechseln. Manche sind wenig über Hanfkorn gross, andere erreichen Kirschkerngrösse, andere werden noch grösser. Einzelne sitzen breit auf, andere wieder sind gestielt und leicht beweglich.

Bei einer gewissen Grösse und bei einer gewissen Entfernung von der natürlichen Ohrgegend machen diese Hyperplasien (Hypergenesien) den Eindruck einer Verdoppelung des Obres, ja sie scheinen in der That in eine. Art von Polyotie überzugehen. Wilde (Ohrenheilkunde, deutsch von Haselberg S. 192) berichtet nach Cassebohm von einem Kinde, das zwei Ohren an der natürlichen Stelle, zwei tiefer am Halse hatte, und wo jedes Schläfenbein zwei Felsentheile gebabt haben soll. Er erwähnt ferner, dass das alte irische Schwein sehr häufig kleine und missgebildete überzählige Ohren, niedrig am Halse sitzend, hat und dass auch Schafe bisweilen 4 Obrén besitzen. Ob die von Heusinger erwähnten Halslappen der Ziegen in diese Kategorie ge- 
hören, weiss ich nicht. Gurlt (Lebrb. der path. Anat. der Haussäugethiere. II. S. 128, 129) erwähnt nur von einem Lamm und von einem Schwein mit Wolfsrachen, dass sie doppelte oder gespaltene Ohren hatten. Offenbar ist diess nicht ganz dasselbe, wie das Frühere. Denn die erste Spaltung des Ohres, wie sie nach der oben erwhihnten Beobachtung von Löffler beim Menschen und nach Gurlt (a. a. 0. S. 115, 460) beim Schwein vorkommt, ist verschieden von der eigentlichen Excessbildung, wie sie Stark berichtet. Für letztere haben wir ein sehr gutes neueres Beispiel von Birkett (Transact. of the Pathol. Society. Lond.1858. Vol. IX. p. 448. Lign. 16): Bei einem wohlgebildeten Mädchen sass jederseits über der Mitte des Sternomastoideus ein grosser, dem Ohrläppchen nach Gestalt und Beschaffenheit äbnlicher Auswuchs, bedeckt mit feinen Härchen; jeder erhielt eine kleine Arterie und besass einen Faserknorpel in seiner Axe, welcher denen des normalen Ohres analog war. Die Ohren selbst waren regelmässig.

Solche Fälle weisen sehr bestimmt auf eine unregelmässige Schliessung der Kiemenspalte hin und sind nicht zu verwechseln mit den, äusserlich ihnen ähnlichen; wo vielmehr die Mundspalte ungewöhnlich erweitert ist. Joh. Fr. Meckel (Path. Anat. I. S.523) erwähnt von einem neugebornen Kalbe totalen Mangel des knöchernen Gaumens, äusserste Kürze des Unterkiefers und eine solche Fortsetzung der Mundspalte durch das Schlafbein und das äussere Ohr, dass dieses in eine grosse obere und eine kleinere untere Hälfte zerrissen war. Hier war also ein einfaches $0 \mathrm{hr}$ in zwei Theile zerlegt, während in den Fällen, die uns zunächst beschäftigen, das Ohr an sich ganz vorhanden ist, aber ausser ihm noch gewisse accessorische Gebilde sich finden.

Allerdings sind auch diese letzteren Fälle in der Regel mit anderweitigen Störungen complicirt. Der nächst einfache Fall scheint mir der zu sein, dass sich gleichzeitig gewisse Anomalien der Kieferknochen zeigen. Diese können entweder die hinteren, oder die vorderen Theile derselben betreffen. Hierhin gehören die schon angeführten Beobachtungen von Allen Thomson über mangelhafte Bildung des Oberkiefers, gespaltenen Gaumen und Fehler der Zahnbildung. Dahin rechne ich ferner einen interes- 
santen Fall, den ich vor einiger Zeit auf meiner Abtheilung beobachtete :

Ein junger Mann in den zwanziger Jahren kam wegen chronischer TonsiltarAngina in das Spital. Bei der Untersuchung des Mundes fand sich ausser einer beträchtlichen, besonders links sehr umfangreichen Hyperplasie der Tonsillen eine bedentende Missbildung des Oberkiefers (Taf. VII. Fig. 7). Derselbe war im Ganzen schmal, wie von der. Seite her zusammengedriickt, und zeigte in seinem vorderen Abschnitt eine zum Theil doppel te Zahnrei he. Vorn standen 3 , sehr verschobene, ungleich grosse Schneidezăhne, und binfer ihnen \& kleinere, sehr unregelmässige Zäbne, deren Deutung im Einzelnen etwas zweifelhalt war, da sebr leicht überzählige darunter sein mochten, und sie zum Theil durch Wülste von Zahnfleisch von einander getrennt waren. Es handelte sich aber unzweifelhaft hauptsächlich um eine Störúng der seitlichen Theile des Zwischenkiefers und der vorderen Enden der beiden Oherkiefer, und zwar in der Gegend, wo sonst die Kieferspalten anftreten (vgl. Taf. VII. Fig. 5). - Weiterhin fand sich an dem linken Ohre eine der beschriebenen ähnliche Missbildung. Vor und über dem Tragus sassen 3 Appendiculargehilde, von denen das oberste die gewöhnliche knopfförmige Gestalt hatte, während das unterste, grössere in eine lángliche Falte auslief, die sich vor dem Ohrlappehen eine Strecke weit heraberstreckte.

Auf die erste Frage nach dem Zustande des Gehörs erwiderte der Kranke, dass er gut höre. Bei genaverer Untersuchung ergab sich freilich, dass die Hörweite links beträchtlich kleiner.war, als rechts; als ich jedoch die linke Tonsille exstirpirt hatte und der Schlund freier geworden war, zeigte sich diese Differenz so gering, dass ich kein besonderes Gewicht darauf legen konnte.

Ich bemerke endlich, dass sich an dem linken Auge eine nach der Angabe des Kranken bis in seine frühe Jugend zurückdatirende, partielle Synechie der lris vorfand, dass aber seit einer vor einigen Jahren vorgẹnommenen Iridectomie das Sehvermögen sich sehr gebessert babe.

Einen Schritt weiter finden wir die verschiedenen Gaumenund Kiefergaumen-Spalten. Die erwähnte Beobachtung von Max Schultze erläutert diesen Fall sehr gut. Ich füge einen anderen bei :

Ein neugebornes Kind (Präparat No. 19 vom Jahre 1864), das mir von der Gebäranstalt der Charité überbracht wurde, zeigte äusserlich bei einem sonst sehr kräftig und voll entwickelten Körper eine doppelte Lippengaumenspalte mit medianer Stellung des Zwischenkiefers, Mikrophthalmie und eine eigenthümliche leistenförmige Hervorragung über der Mitte des seitlich stark zusammengedrückten Stirnbeines. Vor dem linken $0 \mathrm{hr}$ und zwar in der Gegend des Tragus sassen 3 kleine, wenig über Hanfkorn grosse, mit feinen Haaren besetzte Auricularknöpfe, einer über dem anderen (Taf. VII. Fig. 5).

Die weitere Untersuchung lebrte, dass der untere und mittlere Theil des Stirnbeines nicht nur synostotiscb war, sondern auch scheinbar nur einen einzigen and 
zwar centralen Ossificationspunkt (Tuber frontale) besass. Die grosse Fontanelle war noch weit offen, auch die Pfeilnaht an vielen Stellen klaffend und fonticulär. Der Schädel nngemein lang und schmal. Das Gehirn im Ganzen regelmässig gebildet, nur die olfactorii fehlend und um die vorderen Theile der Hirnbasis die Pia mater stark verdickt; dabei leichter Ventricular-Hydrops. Die Optici normal, aber die Augen nicht bloss sehr tief liegend, sondern auch sehr klein. Der äussere Gehörgang, das Trommelfell und die Gehörknöchelchen vorhanden. Unterkiefer normal. - Gleichzeitig fehlte die linke Nabelarterie, beide Hoden lagen noch in der Bauchhöhle, die linke Niere war hydronephrotisch, die rechte eher etwas vergrössert. Brustorgane normal.

Diesem sehr ähnlich ist ein älterer Fall unserer Sammlung (No. 152), nur dass der Sitz des Appendiculargebildes am Ohr ein ziemlich ungewöhnlicher ist:

Unter dem kleinen, ungewöhnlich runden und in der Stirngegend promiDenten Schädel sitzen überaus tief und klein, wie zurückgezogen, die Angen (Mikropthalmie). Doppelte Lippengaumenspalte, der Zwischenkiefer median, an dem sehr beweglichen Septum narium etwas zurückgezogen; jederseits an dem Vorsprunge des Alvelarrandes der Oberkiefer ein rundlicher, weicher Auswachs. Links hinter dem Ohre, ungefähr vor der Spitze des Warzenfortsatzes, ein halberbsengrosser, schwach behaarter Auricularknopf. Das änssere ohr selbst beiderseits normal, die Gehörgänge offen, sehr lang und weit, das Trommelfell sehr weit zurückliegend, in der etwas abgeflacbten Paukenhöhle keine Gehörknöchelchen. Das Grosshirn einfach, ohne Trennung in Hemisphären, Fehlen der Olfactorii and des linken 0 pticus, starker Hydrocephalus der nach vorn noch zusammenhängenden Seitenhirnhöhlen. Unterkiefer ausgebildet.

Wiederum einen Schritt weiter steigern sich die Störungen der Gebörorgane und ihrer Nachbarschaft nach innen hin. In diesen Făllen nimmt der Unterkief er sehr häufig Theil an der Missbildung, jedoch nicht, wie der Oberkiefer, an seinem vorderen Theile, sondern vielmebr an seinem hinteren Umfange, wie die nachstehenden beiden Fälle erläutern werden. Die Beobachtung von Thomson über Mangel des Tuberculum articulare (Proc. auditorius) schliesst sieh hier an, insofern dieser Mangel für das Schläfenbein dasselbe bedeutet, wie die zu beschreibende Mikrognathie für den Unterkiefer.

Ein im Ganzen etwas schwächlich entwickeltes männliches Kind (Präparat No. 20 vom Jahre 1864) zeigt eine ganze Reihe von Störungen der rechten Seite. Nur die Nabelarterie fehlt auf der linken Seite, und die Auricularanbange finden sich beiderseits. Links liegt ein grōsserer und ein hleinerer vor dem Tragus, 
recbts findet sich ein sebr starker, in 4 rundliche Lappen auslaufender Auswuchs gerade in der Incisura intertragica. Das rechte $0 \mathrm{hr}$ ist stark zusammengezogen und zeigt lseine Spur einer Oeffnung zum Gehörgange. Ausserdem sitzt ein etwas über Hanfkorngrosser, Krugeliger Auricularknoten an der Wange, ungefähr über der Gegend des Unterkieferwinkels. - Eine grosse Lippengaumenspalte füllt heinahe die Mittellinie, gehört aber der rechten Seite an; nur sind das Septum narium, der linke Nasenfügel und der Zwischenkiefer stark nacb links hinübergezogen.

An dem rechten Vorderarm hat die Hand eine Stellung, wie bei einer Luxation nach innen. Die Untersuchung ergibt, dass der Radius und das 0 s metacarpi pollicis fehlen, und dass die Handwurzel um das untere Ende der UJoa nach innen verschoben ist.

Das Schädeldach normal. Das Gehirn liess sich nicht mehr untersuchen, da es zu sehr erweicht war. Indess scheinen die Nerven sämmtlich vorhanden zu sein. Auch die innere Ansicht der Felsenbeine bot nichts Auffaliendes dar, als eine gewisse Kärze des äusseren Abscbnittes auf der recliten Seite. Gehörgang, Paukenhöhle, Gehörknöchelchen waren links vorhanden. Rechts dagegen fehlte jede Spur eines Gehörganges und einer Paukenböhle; die ganze Region des Felsenbeins bestand aus einer ungewöhnlich dichten, spongiösen Knochenmasse.

Der Unterkiefer war auf seiner linken Seite normal. Rechts dagegen war er sehr kurz, jedoch ungewöhnlich hoch und an seinem unteren Rande mit einer so stark vorspringenden Leiste verschen, dass es auf den ersten Blick aussah, als wäre hier noch ein besonderer Knochen vorhanden. In der Gegend der Backzähne börte er ganz plötzlich mit einem dicken, abgerundeten Ende auf; dasselbe enthielt noch dicht vor seinem Ende ein grosses Zahnsäckchen. Es fehlten demnach sowobl der Proc. condyloides, als der Proc. coronoides. An dieses Ende schloss sich ein derbes Ligament, welches sich in der Gegend des Tuberculum articulare an das Schläfenbein inserirte; statt eines Tuberculum articulare und eines Processus zygomaticus fand sich nar eine dünne, beweglich angesetzte Knochenspitze. Der Arcus zygomaticus nicht geschlossen; das 0s zygomaticum nach rüehwärts mit einer stumpfen, von pigmentirtem Bindegewebe umgebenen Spitze anslanfend. Um das Unterkiefer-Ligament eine reichliche Fettanbäufung: das Lịgamentum stylohyoideum vollständig vorhanden, innen knorpelig.

Brustorgane normal. In der Bauchhöhle eine feste Synechie zwiscben Gallenblase und Flexura coli hepatica; in Folge dessen die ganze rechte Abtheilung des Colon mit dem Coecum in die Höhe gezogen und das letztere sehr beweglich.

An diesen Fall reiht sich ein, mit noch grösseren Störungen complicirter, der wieder ein älteres Präparat unserer Sammlung (No. 371) betrifft:

Es besteht hier ein Cranium bifidum totale (Acranie) mit Spina bifida occipito-cervicalis. Letztere ist mit totaler Amyelie verbunden, ersteres dagegen mit einer nach links, vorn und abwärts gerichteten Encephalocele, wobei sowohl das 
Auge, als das 0 hr der linken Seite defect sind. Allerdings findet sich noch eine Andeutung des Auges, während das Ohr ohne Oeffnung ist und eine kleine, zusammengezogene Muschel besitzt. Rechts besteht Mikrophthalmie mit sehr tiefer Lage des Bulbus, und vor dem in der Richtung von oben nach unten wie zusammengedrückten Ohre sitzen 3 Auricularanhänge: der grösste, stark erbsengross, dicht vor dem Tragus, der nächstgrösste, fast polypōs gebildete, am Ansatze des Helix, der dritte und kleinste dazwischen. Der grösste enthält ein sehr starkes, bis auf die Fascie durchdringendes Knorpelstück. Ausserdem sitzt an der vorderen Fläche des $0 \mathrm{hrläppchens} \mathrm{ein} \mathrm{kleiner,} \mathrm{kaum} \mathrm{hanfkorngrosser,} \mathrm{kugliger} \mathrm{Auswuchs.}$

Zugleich grosser medianer Wolfsrachen, der jedoch etwas weiter nach rechts hinübergreift. Das Septum narium steht sehr hoch und zurückgezogen; der Zwischenkiefer scheint zu fehlen. Links am oberkiefer ein rundlicher wulstförmiger Vorsprung.

Die weitere Untersuchung zeigt auch hier einen Defect am hinteren Umfange des Unterkiefers auf der rechten Seite. Der Gelenkfortsatz fehlte vollständig.

Die innere Untersuchung des Felsenbeins unterblieb, um das Präparat zu schonen.

Die mitgetheilten Beobachtungen werden genügen, um die Beziehung der Auricularanhänge zu ausgedehnteren Störungen im Gebiete des ersten Kiemenbogens darzuthun. Allerdings finden sich darunter auch solche, wo dieses Gebiet weit uberschritten wurde, und wo nicht bloss die Sehorgane, das Gehirn und der Schädel, sondern auch gewisse Unterleibsorgane, ja sogar die Extremitäten mit betheiligt waren. Allein niemand wird Bedenken tragen, innerhalb einer solchen grösseren Summe von Störungen die näher zusammengehörigen auch zusammenzulegen, und die verschiedenen Störungskreise festzustellen, deren Coincidiren die Gesammtstörung erklärt. Ein solcher Störungskreis wird eben durch die beschriebenen Auricularanhänge bezeichnet, und wenn wir gefunden haben, dass dieselben am bäufigsten dicht vor dem $\mathrm{Ohr}$, in der Gegend des Tragus, nächstdem auf der Wange und am Halse, selten hinter dem Ohr in der Gegend des Warzenfortsatzes vorkommen, so geht daraus hervor, dass die Grenzen des Störungskreises ziemlich umfangreich sind und dass insbesondere, wovon wir ausgingen, die Beobachtung Heusinger's über eine bestimmte Form der Halskiemenfistel noch in diese Grenzen fällt.

Dass die betreffenden Gebilde Auriculargebilde und nicht etwa 
gewöhnliche Naevi sind, ergibt sich nicht bloss aus jhrer sehr eigenthïmlichen Structur, sondern auch aus dem Umstande, dass bei mangelhafter Entwickelung der Ohrmuschel nicht selten eben solche knopfförmige Anbänge an der Stelle der lekzteren erscheinen, als letzte und einzige Andeutungen von ihr.

In dieser Beziehung ist der von Birnbaum musterhaft beschriebene Fall sehr lehrreich. Hier sass an dem knorpeligen Gehörgange nur ein dem Ohrläppchen entsprechendes Hautläppchen und ungefähr $\frac{1}{2}$ Zoll davon nach oben und hinten ein rundlicher Hautwulst mit einer scheinbaren Oeffnung, der knorpelige Theile enthielt. In diesem Falle fehlte der Steigbügel, das ovale Fenster, die Chorda tympani und der Proc. styloides, während von Hammer und Amboss Rudimente, der Annulus tympanicus und das Trommelfell in unvollkommener Form, das runde Fenster und die Tuba Eustachii gut gebildet vorhanden waren. Der Fall ist insofern besonders interessant, als die Störung hauptsächlich das Gebiet des zweiten Kiemenbogens betraf, und dem entsprechend das Gesicht regelmässig gebildet war.

Selbst in den höchsten Graden der Defektbildung, bei Paracephalen, Pseudathoracen, Pseudamorphen, Acormen, Aniden, finden sich an der Stelle der Ohren kugelige oder warzige Auricularknöpfe, welche den supernumerären ganz ähnlich sind. Man vergleiche u. A. Vrolik Beschrijving eeniger merkwaardige misgeboorten. Amst. 1855. Pl. IV. Fig. 1-2. Barkow Beiträge zur path. Entwickelungsgeschichte. Bresi. 1854. Fig. I-II. Abth. II. Bresl. 1856. Taf. I. Fig. I-Il. Mayer dieses Archiv Bd. XXIX. Taf. XIII. Fig. 1:

Diese letzteren Formen sind $z \mathrm{u}$ vergleichen rnit den mangelhaften Nasenbildungen, wie sie bei Cyclopie vorkommen, wo sie jene sonderbaren Rüsselformen darstellen, die zuweilen ibber der Augengegend an der Stirn inserirt sind. Diese gebören dem Stcörungsgebiet des olfactorius an, das freilich in niederem Grade der Stồrung nur jene geringeren Veränderungen erfährt, welche wir in einigen Fällen mitbeschrieben haben; ich meine Mikrophthalmie, prämature Synostose der Stirnbeine, Defekt des Siebbeins, Einfachheit des Grosshirns. 
Sehr natürlich ist es, dass in demselben Falle mehrere Störungskreise benachbarter Embryonaltheile sich vermischen, aber in den meisten Fällen wird es bei einer geordneten Untersuchung noch möglich sein, sie auseinander $z u$ lösen. Ein vorzügliches Beispiel einer solchen Vermischung bietet der von Pokorny (Sitzungsberichte der Wiener Akademie Bd. 46. S. 399) beschriebene Fall von Aprosopie dar. Hier fand sich einerseits die Bildung von Auricularanbängen am Tragus bei Mangel der Paukenhöhjen und der Gehörknöchelchen, andererseits Defekt des Sjebbeins, des Stirnbeins, der Fortsätze des Keilbeins und aller Gesjchtsknochen, der Augen und der Nase, während der äussere Gehörgang, der Annulus tympanicus, das Ligamentum stylohyoideum und das Labyrinth vorhanden waren. Die untere Begrenzung der - ersten Kiemenspalte, der zweite Kiemenbogen war demnacb in seinen Haupttheilen gebildet und nur sein innerstes Ende, der Steigbügel fehlte; die obere Begrenzung, der erste Kiemenbogen, dagegen war bis auf geringe Rudimente defekt, aber die Hauptstörung lag doch in dem Gebiete der vorderen Schädelwirbel und der nächst an sie anstossenden Theile.

Noch charakteristischer sind die unilateralen, aber multiplen Störungen, von denen wir mehrere Beispiele beschrieben haben. Dahin gehören auch die Fälle von Birnbaum und M. Schultze. Hier bietel sich eine sehr beständige und auffallende Erscheinung dar, nämlich das Fehlen dereinen Nabelarterie. Gewiss lässt sich die Frage nicht umgehen, ob dieser Mangel nicht zugleich die anderen Störungen erklärt: Ich glaube aber diese Frage verneinend beantworten zu müssen. Der Mangel der Nabelarterie fällt nämlich nicht immer auf die Seite, wo die Störungen der anderen Theile sich finden, und gewiss ist es leichter, ihn als Coëffekt derselben Ursache zu betrachten, welche auch die anderen Störungen bedingt, als in ihm selbst den Mittelpunkt alleranderen Störungen zu sehen.

Man wird daher im Allgemeinen wohl sicherer gehen, wenn man örtlich wirkende Ursachen voraussetzt. Ich will es dahingeslellt sein lassen, ob hier traumatische, thermische oder welche Ursachen sonst einwirken; die Hauptsache ist, dass es sich über- 


\section{3}

haupt nicht um einfache Defekte handelt, sondern dass die Defekte mehr oder weniger bestimmt auf irritative Prozesse zurückzuführen sind, welche Indurationen, Adbäsionen, narbenartige Retractionen, Synostosen, Hyperostosen und andere Hypergenesien erzeugen. $0 \mathrm{~b}$ man diese Prozesse entzündliche nennen will, ist ziemlich gleichgültig; genug, dass sie keine primär passiven sind, sondern dass der Defekt Folge eines activen oder irritativen Vorganges ist.

Schon bei verschiedenen früheren Gelegenheiten, namentlich bei der Geschichte der Schädelsynostosen (Gesammelte Abhandl. S. 926, 997, Untersucbungen äber die Entwickelung des Schädelgrundes S. 104, Archiv Bd. XIll. S. 351), der cystoiden Degeneration der Nieren (Ges. Abh. S. 872), der multiplen Indurationen und Adhäsionen innerer Organe (Archiv Bd. XXII. S. 432) habe ich auf diese fötalen Irritationen und Enizündungen nachdrücklich hingewiesen. Je weiter ich untersuche, um so mehr überzeuge ich mich von der grossen Bedeutung, welche dieser Gesichtspunkt für die Begründung einer auf genetischem Grunde ruhenden Teratologie hat.

So lange man die Gaumen - und Kieferspalten bloss als Defekte auffasst, so lange kommt man nicht an das Wesen derselben und noch weniger an den Mittelpunkt des Störungskreises, um den es sich hier handelt. Wenn die Gaumenbeine nicht aneinander treten, wenn die Oberkiefer die $\mathrm{Z}$ wischenkiefer nicht erreichen, so muss etwas vorhanden sein, was sie zurückhält. Unter Umständen kann diess ein Vorgang sein, der an ihrem vorderen Umfange verläuft, wie wir es am deutlichsten in jenen Fällen sehen, wo eine Synechie mit den Eihäuten, insbesondere mit der Placenta foetalis sich bildet und theils die adbäsiven Bänder, theils die Eihäute selbst sich zwischen und um die Enden der einander entgegenwachsenden Knochen legen und sowohl dadurch, als durch den Zug, den sie ausüben, die Knochen getrennt balten. Anderemal aber liegt der Grund gewiss nicht vorn, sondern hinten, und diess wird sicherlich jedesmal der Fall sein, wenn Anomalien des äusseren oder mittleren Ohres oder gar Anomalien des Unterkieferastes zugegen sind, wie ich sie oben in mehreren Fällen genauer geschil- 
dert habe. Es gibt also Formen der Gaumenspalte und des Wolfsrachens, welche aus frühzeitigen, insbesondere irritativen Störungen des ersten Kiemenbogens hervorgehen, und welche mit bestimmten Missbildungen des äusseren und mittleren ohres eine gemeinschaftliche Quelle haben.

Dabei ist es aber besonders bemerkenswerth, dass das innere Ohr unmittelbar nicht dabei betheiligt ist, dass im Gegentheil der Hörnerv, das Labyrinth u. s. w. ganz normal sein können. Diess haben sowohl Toynbee und Pokorny, als namentlich mit grosser Genauigkeit Lucae nachgewiesen. In dieser Beziehung besteht eine durchgreifende Verschiedenheit zwischen den Zuständen des Acusticus und des Olfactorius, welche gewiss für die Kritik der Störungskreise von grösster Bedeutung ist.

\section{IX.}

\section{Das Hygroma llyo-epiglotticum.}

Von Prof. Dr. H. Luschka in Tübingen.

Indem wir mit dem Nachfolgenden die Aufmerksamkeit auf das Vorkommen einer serösen Balggesehwulst im Bereiche der vorderen Seite des Kehldeckels zu lenken suchen, können wir es um so weniger unterlassen, über die hier obwaltenden normalen Verhältnisse einige Erőrterungen vorauszuschicken, als dieselben noch keineswegs mit einer der praktischen Wichtigkeit des Gegenstandes gemässen Genauigkeit dargelegt worden sind.

Die hier zu erwägende Frage betrifft zunächst die räumlichen Beziehungen desjenigen Theiles der vorderen Fläche der Cartilago epiglottidis, welcher mit der Schleimbaut nicht in Berübrung steht. Während die nach pückwärts schauende Seite des Kehldeckels in ihrer ganzen Länge und Breite frei theils der Höhle des Schlund- 